\title{
Un siècle de publications de la Société d'histoire de la révolution de 1848. Politique éditoriale et évolution des thèmes de recherche.
}

\section{Raymond Huard}

\section{OpenEdition}

\section{Journals}

Édition électronique

URL : http://journals.openedition.org/rh19/947

DOI : $10.4000 /$ rh 19.947

ISSN : 1777-5329

\section{Éditeur}

La Société de 1848

\section{Édition imprimée}

Date de publication : 1 décembre 2005

ISSN : 1265-1354

\section{Référence électronique}

Raymond Huard, «Un siècle de publications de la Société d'histoire de la révolution de 1848. Politique éditoriale et évolution des thèmes de recherche. », Revue d'histoire du XIXe siècle [En ligne], 31 | 2005, mis en ligne le 18 février 2006, consulté le 22 avril 2019. URL : http://journals.openedition.org/ rh19/947 ; DOl : 10.4000/rh19.947

Ce document a été généré automatiquement le 22 avril 2019

Tous droits réservés 


\title{
Un siècle de publications de la Société d'histoire de la révolution de 1848. Politique éditoriale et évolution des thèmes de recherche.
}

\author{
Raymond Huard
}

1 C'est en 1906 que notre société, deux ans après sa création, décida de procéder à des publications qui s'ajouteraient à son Bulletin. Avec quelques discontinuités, cet effort s'est poursuivi jusqu'à nos jours. Il nous est apparu intéressant de jeter un regard d'ensemble sur le résultat séculaire de cet effort spécifique en retenant deux angles d'approche qui s'imposaient, à vrai dire, de façon assez évidente. Le premier porte sur l'évolution de la politique éditoriale sous tous ses aspects : quels ont été les types de publication retenus (documents, études, actes de colloque, numéros spéciaux de revues); quel en a été le rythme et quelles perturbations les événements politiques majeurs (les guerres mondiales par exemple) ont-elles pu apporter à cet effort? D'autre part, avec quels partenaires ces publications ont-elles été faites (éditeurs, autres sociétés savantes), où l'argent nécessaire a-t-il été trouvé et comment une association qui n'a eu qu'occasionnellement un support institutionnel universitaire officiel a-t-elle pu se débrouiller - le mot n'est pas excessif pour procéder à celles-ci? Quels ont été nos échecs et les projets avortés? Quelle influence enfin l'évolution générale des formes de la recherche scientifique au cours du siècle écoulé a-t-elle eue sur la politique de publication de notre société ? Ce premier aspect s'inscrit dans les recherches sur l'histoire matérielle des sciences humaines dans notre pays.

2 Le second aspect de notre étude qui s'imposait également, et même de façon plus évidente encore, c'était de percevoir à travers les publications qui, à défaut de prendre en compte tout ce qu'avait publié la société dans sa revue, pouvaient servir de bon révélateur, comment les centres d'intérêt, les thèmes de recherche, avaient évolué, en fonction de l'évolution générale de la recherche historique, mais aussi parfois de la 
conjoncture politico-sociale ou d'occasions plus circonstancielles comme les anniversaires ou comme l'influence de tel président. Ces approches, on le saisit d'emblée, seront avant tout descriptives, parce qu'en dehors de l'influence des personnalités que l'on peut parfois repérer, ce sont le plus souvent des facteurs généraux qui agissent. Sous ces deux angles d'approche, ce bilan peut nous fournir des réflexions utiles pour notre propre travail aujourd'hui ${ }^{1}$.

3 Encore faut-il s'entendre d'abord sur ce qu'on appellera publications de la société. Pour définir celles-ci, on a choisi une option assez large contenant deux rubriques. En premier lieu, les publications proprement dites, bien distinctes de la revue, et considérées comme telles par la société, au nombre de 35 volumes en y incluant les actes du dernier colloque sur 1851. Et en second lieu, les numéros thématiques de la revue, apparus plus tardivement au nombre de 17 jusqu'à la date de cette commémoration, au total cinquante-deux titres recensés ci-dessous et dont une partie (les numéros 1 à 24) a d'ailleurs été regroupée sous le titre : Bibliothèque de la révolution de $1848{ }^{2}$.

4 La chronologie de ces parutions est assez simple. De 1906 à la guerre de 1939, l'effort de publication qui reste limité (14 ouvrages publiés entre 1906 et 1938) révèle une certaine continuité à la fois dans le contenu et la forme, malgré la césure bien marquée de la guerre de 1914-18. La Deuxième guerre mondiale introduit une nette rupture. Pour des raisons diverses, la société tâtonne longtemps avant d'aboutir à la formule plus durable qu'elle finira par adopter au cours des années 1980. Pendant ces cent ans, la politique éditoriale a sensiblement évolué et c'est d'abord celle-ci que nous allons examiner.

5 C'est à l'Assemblée générale du 24 février 1906 que George Renard annonça, après en avoir fait approuver l'idée par le comité directeur le 30 janvier: «Notre intention, que nous vous soumettons, serait d'employer les sommes que nos dépenses modestes laisseront en caisse, à commencer la publication de documents inédits présentant un intérêt général ${ }^{3}$; et il proposa de commencer par les procès-verbaux du Comité du travail de l'Assemblée constituante. À l'Assemblée générale de 1907, l'entreprise avait progressé et les conditions de l'édition furent précisées: l'éditeur serait la Société nouvelle de librairie et d'édition qui distribuait déjà le Bulletin de la société de 1848, ainsi d'ailleurs que la revue La Révolution française. La société de 1848 achèterait à l'éditeur 200 ouvrages au moins avec un rabais de $25 \%$ et les céderait à ses sociétaires à un prix à déterminer (ce sera à $50 \%$ du prix coûtant). Le reste, probablement fort peu de choses, serait vendu au public ${ }^{4}$. Dès 1907, alors que le premier volume n'était pas encore paru, un second était mis en chantier, à l'initiative d'Alphonse Marius Gossez, petit-fils par sa mère du quarante-huitard lillois Alphonse Bianchi, et alors professeur à Montivilliers en SeineInférieure. Il s'agissait des Mémoires de l'ouvrier François Leblanc, adjoint au maire de Monville, dans le même département. Les ouvrages feraient partie - c'est l'Assemblée générale de 1907 qui le décida - d'une collection intitulée «Bibliothèque de 1848 " $^{5}$. L'idée n'est pas nouvelle car à l'époque, les Bibliothèques de ce genre fleurissent autour de la Revue historique, de la Revue d'Histoire moderne et de la société d'études robespierristes. Au départ, les intentions étaient donc claires : il s'agissait de publier des documents inédits, souci majeur des chercheurs à l'époque, qu'ils travaillent sur la Révolution française ou celle de 1848, documents édités sous l'égide de la société et inclus dans une collection, la Bibliothèque de 1848.

6 Très vite cependant, ces intentions furent détournées, au moins partiellement, et la Société se mit à patronner (plutôt que de les publier elle-même) des travaux de recherche, et non seulement des documents, qu'elle inclut dans sa Bibliothèque de 1848 
sans que cela fût forcément mentionné sur les ouvrages eux-mêmes. Ce fut le cas du livre d'Hubert Bourgin sur Considerant ${ }^{6}$ dont les chapitres avaient déjà paru dans le Bulletin, puis, en 1910, de la thèse de François Dutacq sur l'histoire politique de Lyon pendant la Révolution, thèse éditée chez Cornély devenu éditeur de la société en 1909 en même temps qu'il avait pris la suite de la Société nouvelle. Pour ce dernier ouvrage, la société s'était contentée de donner une subvention de 100 francs. L'intention primitive n'était cependant pas abandonnée. La preuve en est le projet, envisagé avec faveur par la direction de la société à partir de 1909, d'éditer le Mémorial d'Hippolyte Carnot dans lequel celui-ci évoquait ses souvenirs des années 1848 et 1849. Le document avait été confié à la société par Adolphe Carnot et la publication devait être préparée par Georges Renard sous le contrôle d'Ernest Denis ${ }^{7}$. Mais la parution du Mémorial toujours annoncée comme imminente, fut sans cesse repoussée. « Il aurait déjà paru - précisait le secrétaire général Hippolyte Moysset en 1911 - si le Comité n'avait jugé utile de le faire précéder d'une introduction historique qui a nécessité d'assez longues recherches. Elle fera voir par le menu détail psychologique la formation intellectuelle et politique d'une homme représentatif de l'idéal de 1848 et marquera en traits rapides mais précis la ligne de tradition républicaine qui relie le grand Lazare Carnot au président Sadi Carnot et à notre vénéré président d'honneur $\mathrm{M}$. Adolphe Carnot ${ }^{8}$.

Excès de souci érudit ou autre facteur que nous ignorons? Le Mémorial ne parut pas - à l'exception d'un extrait publié dans la revue ${ }^{9}$ - et l'on peut se demander si le manuscrit en est toujours disponible quelque part. En attendant, la société avait publié trois volumes dans la seule année 1911: l'ouvrage d'Hippolyte Monin sur Bancel, la présentation de l'œuvre du poète allemand Georges Herwegh par Victor Fleury, et le livre de Robert Pimienta sur la propagande bonapartiste en 1848.

8 La guerre de 1914 interrompit naturellement cet effort et la reprise, très lente, se dessina seulement à partir de 1926. Le premier volume de l'après-guerre préparé par Félix Ponteil n'était qu'un simple tiré à part de la revue sur La crise alimentaire dans le Bas-Rhin en 1847. Le mouvement s'accéléra à partir des années 1930 et la société publia six ouvrages entre 1932 et 1938. C'étaient des publications modestes (moins de cent pages le plus souvent), portant avant tout sur l'histoire politique et sociale. Remarquons que les documents avaient à nouveau une nette prépondérance (quatre ouvrages sur six), en particulier grâce aux efforts de l'infatigable Alphonse-Marius Gossez. Frédéric Rieder, employé de Cornély, ayant repris la maison d'édition en $1913^{10}$, c'est désormais lui qui éditait nos travaux. Au total, à la veille de la Deuxième guerre mondiale, la bibliothèque de 1848 comprenait quatorze ouvrages d'importance très variable (d'une trentaine de pages à plus de 400) dont la moitié environ présentaient des documents.

9 Avec la guerre, les publications et d'une façon générale, l'activité de la société cessèrent à nouveau. En 1939, la société ne comptait plus d'ailleurs que 110 membres. À la Libération, elle reprit vie dès 1945 sous la direction du même président qu'avant la guerre, Justin Godart, de tendance radicale avec un nouveau secrétaire général, Emile Tersen, professeur de khâgne à Louis le Grand, qui représentait la mouvance communiste ${ }^{11}$. À cette date, les difficultés d'édition étaient grandes par suite du manque de fonds et de papier. Le moment était pourtant décisif car on commençait à envisager la célébration du centenaire de 1848. De celui-ci, notre société fut partie prenante, certes, et même de façon très active. Mais la commémoration ne se fit pas à sa seule initiative ${ }^{12}$. C'est en effet une commission officielle nommée le 8 février 1947 qui fut chargée de la préparer, commission présidée par Edouard Depreux, ministre de l'Éducation nationale, et qui 
donna naissance elle-même à plusieurs commissions spécialisées, soit pour la préparation d'une exposition qui se tint à la Bibliothèque nationale, soit pour la publication de documents car ceux-ci n'étaient pas oubliés (de cette commission, le président fut Charles-Hippolyte Pouthas), soit pour le congrès du centenaire qui eut lieu du 30 mars au 4 avril 1948 et qui se tint sous l'égide de trois organismes: notre société, la société d'histoire moderne et le comité des travaux historiques et scientifiques. À l'occasion du centenaire, la contribution de la société aux publications, contribution qui ne fut que partielle, revêtit trois aspects. D'abord, et sous l'égide du comité créé à cet effet, la publication d'un volume de documents, les Procès-verbaux du gouvernement provisoire et de la commission du pouvoir exécutif, préparés par Albert Soboul, qui parurent en $1950^{13}$. Un second volume prévu qui aurait rassemblé les Actes diplomatiques du gouvernement provisoire et de la commission du pouvoir exécutif ne semble pas être venu à terme. À ces publications, il faut ajouter les Actes du Congrès historique du centenaire de la révolution de 1848, qui furent publiés sous l'égide du Comité français des sciences historiques. C'est dans cet ouvrage que figure la célèbre communication d'Ernest Labrousse, «1789, 1830, 1848, comment naissent les révolutions ${ }^{14}$. Enfin, dans le cadre de cette commémoration, les Presses universitaires de France, qui avaient entre-temps absorbé les éditions Rieder, entreprirent de publier une collection de petits ouvrages (environ 80 pages) de bonne vulgarisation, dont la direction fut assumée, d'après Rémi Gossez, par Emile Tersen ${ }^{15}$. Vingt-trois ouvrages furent ainsi publiés en 1948 sur des sujets très divers, mais qui portaient surtout sur l'année 1848. Il s'agissait de biographies (Blanqui, Louis Blanc, Ledru-Rollin, Raspail, Lamartine, Buchez, Hippolyte Carnot), de récits d'épisodes marquants (les journées de Février, l'avènement du suffrage universel, l'abolition de l'esclavage, Lyon en 1848 - sous la plume du président de la société, Justin Godart), d'études sur les théoriciens ou publicistes sociaux de l'époque, tels que Cabet, Marx, les fouriéristes, Eugène Sue (ce dernier par Pierre Chaunu alors âgé de 25 ans et dont c'est une des premières œuvres), Lamennais présenté par René Rémond, jeune historien lui aussi. Emile Tersen avait traité de la politique extérieure du gouvernement provisoire. Les femmes de 1848 n'avaient pas été oubliées grâce à une étude d'Edith Thomas, conservateur aux Archives nationales, ni non plus le personnage du "Quarante-huitard ", traité par Jean Cassou. Malgré leur incontestable qualité, ces publications n'eurent pas, semble-t-il, le succès espéré. Si à l'occasion du centenaire, notre société fut donc présente sur tous les terrains (colloque, exposition, publications savantes, publications populaires) en particulier grâce à l'activité considérable de son secrétaire général Emile Tersen, sa contribution, dans le domaine des publications, est cependant partagée avec d'autres organismes dans des proportions qu'il est assez difficile de déterminer. Il n'en est pas moins vrai que cette commémoration accapara sans doute l'activité des dirigeants de la société entre 1946 et 1949.

10 Après cet épisode, la société dut adapter son action aux circonstances nouvelles de la guerre froide qui divisait profondément la gauche. Elle avait déjà élargi son champ d'action en dénommant sa revue, dès 1946, 1848 et les révolutions du XIXe siècle. En 1949, elle l'élargit encore, sans doute sous l'influence de son aile gauche, en l'appelant 1848, revue des révolutions contemporaines et en annonçant sa volonté d'ouvrir plus largement son champ d'étude, et dans le temps, et vers les pays étrangers, ce qui suscita d'ailleurs les réserves et le retrait de Charles-Hippolyte Pouthas. Justin Godart, très âgé, quitta la présidence en 1953 - il devait mourir peu après - et fut remplacé d'abord par Georges Bourgin, de 1953 à 1955, puis par Ernest Labrousse, professeur à la Sorbonne depuis 1945. La revue vivait difficilement. La suppression de l'aide du CNRS lui fut fatale et elle dut 
arrêter sa publication à la fin de 1951. Le CNRS suggéra de la remplacer par « un recueil, si possible annuel, d'histoire comparée, autour d'un thème central, composé d'études spécialisées, avec une collaboration internationale ${ }^{16}$. Cette orientation n'était sans doute pas sans intérêt sur le plan scientifique, mais elle était trop ambitieuse pour une société de bénévoles, munie de peu de moyens comme la nôtre, et surtout, mais on s'en aperçut plus tard, elle minait l'existence même de la société. Les publications reprirent certes en 1953 et 1954, notamment grâce à l'activité de Rémi Gossez, sous la forme d'un volume d'Études, au départ annuel, qui était en fait composé d'articles variés dont le seul lien était de traiter de la Seconde République. L'on passa ensuite à des recueils thématiques qui devaient en principe prolonger et élargir la conférence faite à l'Assemblée générale. Ils portèrent sur l'armée en 1955, sur la crise économique de 1846-51 en 1956, sur le choléra en 1958, sur les élections de 1869 en 1960. Mais il fut impossible de tenir le rythme annuel qui passa d'abord à deux, puis trois ans. Le souci de présenter des documents demeurait présent puisque dans les Études de 1953, Rémi Gossez publiait et commentait une carte nationale des mouvements liés à l'impôt des quarantecinq centimes et que le volume sur les élections de 1869 contenait plusieurs cartes très élaborées des résultats électoraux. La société était désormais son propre éditeur, ce qui rendait la tâche plus difficile, jusqu'à ce qu'elle ait trouvé, en 1960, le concours de la librairie Marcel Rivière. Quant à la diffusion, elle était assurée, dans des conditions qui restaient confidentielles, par la Librairie du labyrinthe, rue Cujas, qui était la propriété du trésorier de la société, Charles Lecompt ${ }^{17}$. Les deux derniers volumes, La presse ouvrière, dirigé par Jacques Godechot et Rémi Gossez, en 1966, et Les ouvriers de Paris, premier tome de la thèse de Rémi Gossez, en 1968, furent financés partiellement par ce dernier qui dut, pour publier sa thèse, contracter un emprunt au Crédit municipal ${ }^{18}$. Notons enfin la sortie, en 1957, d'une table, établie par Lise Dubief des publications de la société depuis l'origine, table qui est toujours un instrument de travail très utile, malheureusement devenu rarissime. En 1970, la société dont l'existence devenait de plus en plus hypothétique puisque les adhérents ne recevant plus chaque année de pâture minimale tendaient à la déserter, n'avait donc plus aucun moyen de publication, ni de diffusion car entre temps, la Librairie du labyrinthe avait fermé ses portes. C'est pourquoi les deux volumes suivants qui étaient annoncés, pourtant très neufs par leur thématique, l'un proposé par Jean Dautry sur «Chômage et bas-fonds au XIX siècle ", l'autre, par Louis Chevalier sur "Les criminels dans les sociétés occidentales au XIX ${ }^{\mathrm{e}}$ siècle ", restèrent à l'état de projet ${ }^{19}$. Avec Les Ouvriers de Paris, la Bibliothèque de 1848 avait donc pris fin.

11 Il fallut attendre 1975 pour que la société émerge de sa léthargie, grâce la conjonction des efforts de Maurice Agulhon, nouveau président, de Rémi Gossez, Michelle Perrot, Albert Soboul et Philippe Vigier. La question des publications fut immédiatement posée et la démarche envisagée au départ fut à peu près la même que celle qui avait été adoptée en 1953, c'est-à-dire d'abord un ou plusieurs numéros de Varia, puis des volumes thématiques regroupant autour de la conférence annuelle, un ensemble d'études sur le même sujet ${ }^{20}$. Elle se heurta aux mêmes difficultés qu'auparavant. Certes la société put bénéficier grâce à Albert Soboul de "l'hospitalité » (selon le mot de Maurice Agulhon) ${ }^{21}$, des Annales historiques de la Révolution française qui avaient accepté de publier un numéro par an, préparé par nos soins. Un numéro de Varia parut donc dans ces conditions en 1975. Le suivant, un numéro à thème fut consacré sur la proposition de Michelle Perrot, à "la prison" et son histoire fut très originale ${ }^{22}$. Il prolongeait une communication prononcée par l'intéressée à l'assemblée générale de 1976. Outre le moment postsoixante-huitard, l'ouvrage de Michel Foucault, Surveiller et punir, paru en 1975, avait 
attiré l'attention des historiens sur la question. Le numéro, qui parut sans titre, faute d'accord sur ce point, comprenait, outre la conférence de Michelle Perrot, sur «1848, révolution et prisons ", un certain nombre d'études sur ce sujet (Nicole Castan, Catherine Duprat, Jean-Jacques Darmon, André Zysberg) ainsi qu'une lecture critique, très incisive, du livre de Foucault, par Jacques Léonard, spécialiste d'histoire de la médecine, trop tôt disparu ${ }^{23}$. Ce numéro eut un certain succès et, pour une fois, fut vite épuisé. Il fut communiqué par Michelle Perrot, à Michel Foucault qui proposa de répondre à Jacques Léonard et de tenir une table ronde au cours de laquelle se confronteraient philosophes et historiens. La réponse de Michel Foucault à Jacques Léonard, sous le titre, «La poussière et le nuage » fut très acide ${ }^{24}$. La table ronde rassembla des philosophes (notamment François. Ewald) et des historiens (dont Michelle Perrot et Maurice Agulhon) dans une atmosphère courtoise, mais tendue. Elle fut enregistrée, puis décryptée et réécrite pour une publication au Seuil, avec l'accord de Michel Winock. Les historiens durent lutter contre l'éditeur pour garder dans l'ouvrage une place honorable. Le désaccord perceptible entre historiens et philosophes fut consacré par une double postface de Michel Foucault et Maurice Agulhon. L'ouvrage final parut sous le titre L'impossible prison, en 1980. Il représente un cas unique dans l'histoire de notre société.

La collaboration entre la Société des études robespierristes et la nôtre, inaugurée avec ces deux numéros, ne fut que temporaire. Elle suscitait des oppositions au sein de la première dont elle élargissait de façon excessive le champ d'études ${ }^{25}$ et nous imposait un rythme de parution auquel sans doute nous n'étions pas préparés à cette date. Après un essai de collaboration avec Romantisme, l'on en revint donc à des publications séparées. Le premier volume de celles-ci prolongeait une conférence de Marcel Emerit sur les saint-simoniens à l'Assemblée générale de 1977 et fut consacré aux Utopismes sociaux. Il fut préparé sous la direction de Jacques Valette, professeur à l'Université de Poitiers, qui avait consacré sa thèse au fouriériste Jules Duval ${ }^{26}$. Le volume final que le CDU-SEDES accepta de publier comprenait une très longue introduction de JacquesValette (près de $40 \%$ du volume), puis des communications diverses (dont celle de Marcel Emerit) sur les socialismes utopiques. Le deuxième volume, Blanqui et les blanquistes, marqua en revanche l'entrée de la société dans une ère nouvelle, celle des colloques. Le colloque Blanqui préparé lui aussi par Maurice Agulhon, président, et par Pierre Ayçoberry et Jacques Valette, secrétaires généraux successifs, se déroula, faute de moyens appropriés, de façon un peu " confidentielle ${ }^{27}$ selon l'expression de Maurice Agulhon, en 1981, pour le centième anniversaire de la mort de Blanqui, mais la publication n'intervint qu'en 1986, par suite des lenteurs du même éditeur. Vers cette date donc, les prémisses avaient été posées pour un redémarrage des publications, mais elles demeuraient assez précaires, l'éditeur traînait les pieds, la société n'avait pu encore se lancer dans la publication d'une revue.

13 Le début des années 1980 voit s'ouvrir une phase de renouvellement de la recherche universitaire marquée d'abord par la multiplication progressive des colloques qui est un phénomène beaucoup plus général et correspond à une certaine mutation de la recherche liée à l'augmentation sensible du nombre de jeunes chercheurs et à leur regroupement dans des centres de recherche spécialisés. Le renouvellement progressif des moyens d'impression des publications grâce à l'informatique apporte de nouvelles facilités et les sources de financement se diversifient (Universités, CNRS, CNL, ministères divers, autres organismes). Philippe Vigier, nouveau président, avait groupé autour de lui de jeunes historiens très dynamiques, Alain Faure, secrétaire général de la société, Jean-Claude Caron, Jean-Yves Mollier, qui firent beaucoup pour démultiplier l'activité de la société et 
accroître le nombre de ses adhérents. La politique de publication fut reprise et systématisée, d'abord par la reparution de la revue en 1985 sous la direction de JeanClaude Caron, à raison d'un numéro par an, édité par les soins de la société elle-même, puis par l'édition de colloques désormais périodiques. Alors que le CDU-SEDES était de plus en plus réticent à poursuivre sa collaboration éditoriale avec notre société, l'existence d'une jeune maison d'édition Créaphis fondée en 1982 par Pierre Gaudin et Claire Reverchon, docteurs en histoire et dont l'orientation éditoriale, au départ tournée vers l'étude de la pierre, prenait de plus en plus en compte l'histoire sociale et politique, fournissait une solution de rechange tout à fait opportune ${ }^{28}$. Cette politique éditoriale se diversifie encore un peu plus quand à partir de 1989, apparaissent les numéros à thème de la revue dont le premier coordonné par Jean-Claude Caron porta sur le sujet: « Histoire de centenaires ou le devenir des Révolutions. Contribution à l'histoire des centenaires des révolutions de 1830, 1848, 1870 et 1871 en France et en Europe ». L'édition de numéros spéciaux alternant avec des Varia fut rendue plus facile quand sous l'impulsion d'Alain Corbin, son président, la revue passa à deux numéros par an en 1996. Ainsi, de 1997 à 2004 sont publiés (parfois en collaboration) huit colloques et dix-sept numéros à thème sur des sujets très divers qu'on abordera plus loin.

En matière de politique éditoriale, le bilan des vingt dernières années est très riche, mais il traduit aussi des infléchissements. La première constatation, c'est un accroissement significatif du nombre de publications qui alourdit sensiblement le travail des bénévoles qui administrent la société et cet alourdissement a atteint actuellement ses limites. Autre constatation, l'effacement progressif de la publication de documents. Celle-ci devient ou bien le fait d'éditeurs privés, ou bien a été remplacée dans les centres de recherche universitaires par de grandes enquêtes collectives de caractère prosopographique (notamment sur le personnel politique, maires, conseillers généraux, députés et sénateurs par exemple, ou sur les militants ouvriers dans le cadre du Maitron). Enfin le contenu même des ouvrages publiés, qu'il s'agisse des numéros spéciaux ou des colloques, s'inscrit dans une forme dominante, c'est à dire le rassemblement d'un faisceau d'études venant d'auteurs divers sur une question, plutôt que l'édition d'un ouvrage à auteur unique comme c'était le cas dans les premières années de la société. La société de 1848 n'est pas originale à cet égard; elle a suivi le mouvement général de la recherche universitaire.

15 En même temps les thèmes étudiés se sont modifiés de façon sensible et c'est le second aspect de notre étude.

16 L'évolution des thèmes abordés est bien perceptible depuis l'origine et assez significative. Elle reflète de façon évidente les grandes tendances historiographiques qui se sont imposées successivement ainsi que l'influence sur nos thèmes d'étude, de la conjoncture politico-sociale et idéologique générale, l'équation personnelle de nos présidents successifs et aussi l'évolution générale de notre société où l'accent militant s'est affaibli avec le temps au profit de l'aspect savant.

17 Ce qui dominait, sans toutefois être exclusif, dans les années antérieures à la guerre de 1939-1945 (car la guerre de 1914 ne semble pas avoir apporté d'inflexion décisive), c'étaient sous l'influence sans doute de Georges Renard d'abord et d'Alphonse-Marius Gossez, les problèmes du monde ouvrier sur lesquels portent à peu près la moitié des publications et en particulier les documents originaux publiés par la société. Au témoignage des principaux responsables, Hippolyte Monin, Hippolyte Moysset et Georges Renard, le premier document publié, les Procès verbaux du Comité du travail de l'Assemblée 
constituante avait été choisi « parce que la question du capital et du travail fut la question maitresse du moment, celle qui donne encore aux événements d'alors leur caractère et leur sens particulier " ${ }^{29}$. D'autre part l'attention se porte surtout sur la France et l'intérêt pour l'étranger est faible, puisque, en trente ans, une seule publication, concernant le poète allemand George Herwegh, lui est consacrée. La période suivante qui va de l'aprèsguerre à 1968 se caractérise au contraire par une très grande ouverture, une très grande diversité de thèmes sans aucune prédominance de l'histoire économique et sociale, même si le beau volume édité sous la direction d'Ernest Labrousse sur la crise de 1846 à 1851 a fait date sur le plan scientifique. Et ceci invite à questionner ou au moins à nuancer l'idée parfois avancée de la domination de l'histoire économique et sociale, plus ou moins liée elle-même à l'influence du marxisme sur la vie intellectuelle française pendant cette période. Dans ses publications propres, telles que je les ai définies au départ, notre société pendant cette période a peu traité des questions économiques et n'a consacré qu'un seul article à Marx ${ }^{30}$, aucun à Engels, ni à d'autres marxistes. Pendant les années 1950 et 1960, le spectre offert par les publications est au contraire très large : armée, élections et droit de suffrage, presse ouvrière, épidémies abordées pour la première fois avec un numéro sur le choléra de 1832 dirigé par Louis Chevalier. De même l'ouverture des publications sur le monde (et en particulier sur l'Europe) est beaucoup plus marquée notamment dans les deux volumes sur le choléra et la presse ouvrière auxquels participèrent des chercheurs anglo-saxons, allemands, hongrois et russes. Rappelons que Pierre Renouvin au Congrès du centenaire avait donné une communication sur "l'idée d'États-Unis d'Europe pendant la crise de $1848{ }^{31}$ et que ce colloque fit une large place aux historiens étrangers, notamment de l'Europe de l'Est (Hongrie, Pologne, Yougoslavie, Tchécoslovaquie).

18 La phase ultérieure qui correspond au redémarrage de la société après 1975 sous les directions successives de Maurice Agulhon et Philippe Vigier subit évidemment l'influence de l'atmosphère post-soixante-huitarde. Si l'accent est mis sur le socialisme non marxiste à l'occasion du colloque sur les utopismes sociaux ou du colloque Blanqui, ce n'était pas très nouveau dans une société qui a toujours consacré beaucoup plus d'attention au socialisme non marxiste qu'à Marx. Plus neuve est l'attention portée aux différents aspects de la contrainte d'État (prison, répression, maintien de l'ordre), avec les numéros spéciaux ou actes de colloque consacrés à ces sujets. Mais notre société, comme le montrent à la fois ses publications et le débat avec Foucault, s'est attachée à les aborder d'un point de vue strictement historique et a ainsi contribué à l'exploration de ce champ de recherche neuf et très riche.

Restent les quinze dernières années que beaucoup d'entre nous ont vécues, qui ont été marquées notamment par les présidences d'Alain Corbin (qui succéda à Philippe Vigier) et de Jean-Luc Mayaud. Quelques termes pourraient résumer notre évolution, très complexe, si on la considère du point de vue des publications: élargissement, diversification des thèmes, volonté comparatiste plus affirmée. Ces évolutions correspondent d'abord à la volonté manifestée par la société d'étendre son action à l'ensemble du XIX ${ }^{\mathrm{e}}$ siècle et même parfois au-delà. Elle tient aussi au fait que tout en gardant un certain nombre d'orientations fondamentales, elle a été entraînée - et c'est normal - dans la diversification très marquée des recherches historiques qui a caractérisé ces années et qui a abouti à ce qu'on a pu appeler parfois un véritable éclatement de l'histoire. Ces nouveaux champs, la société les a d'ailleurs plus ou moins bien abordés. 

de son effacement momentané, les grands anniversaires du 4 septembre 1870 et de la Commune, ne pouvait ignorer évidemment ni le bicentenaire de la Révolution française dont les effets sur le XIX ${ }^{e}$ siècle sont majeurs (Ernest Labrousse le rappelait dès les années 1950) ${ }^{32}$, ni le cent cinquantenaire de 1848 , ni celui de décembre 1851 qui donnèrent chacun lieu à un grand colloque et en ce qui concerne les deux derniers événements, à plusieurs numéros spéciaux très nourris. La vision des événements a pu être ainsi élargie de façon sensible. Le même élargissement est constatable dans le traitement d'un sujet en apparence très classique et très dix-neuviémiste, «la barricade » où le recours à une vision comparatiste du $\mathrm{XVI}^{\text {e }}$ siècle à nos jours, et le passage à l'échelle européenne, ainsi que la diversification des approches ont donné au sujet une ampleur et un intérêt nouveaux.

D'autres thèmes, faiblement abordés jusque là ou tout à fait neufs, ont fait leur apparition : les femmes, l'Europe, la jeunesse. L'histoire culturelle a été abordée de façon un peu plus systématique. Revenons sur quelques-uns de ces points et d'abord sur la question féminine. La société de 1848 n'avait jamais vraiment négligé les femmes, et ceci dès l'origine, puisque pour ne prendre que quelques exemples, la revue a consacré dès 1907-1908 plusieurs articles à Jeanne Deroin, sous la plume d'Adrien Ranvier ${ }^{33}$, qu'en 1949, Jean Bossu y traitait des républicaines du Second Empire ${ }^{34}$, et Eugène Schulkind la même année, des femmes de la Commune ${ }^{35}$, que, dans les Études de 1953, Edith Thomas publiait des lettres de Pauline Roland ${ }^{36}$. Dans le volume consacrée à la presse ouvrière publié en 1966, Evelyne Sullerot donnait un article remarquable sur « Journaux féminins et lutte ouvrière 1848-1849 ${ }^{37}$. Evidemment, pendant toute cette période, l'attention se portait davantage sur les femmes militantes que sur les femmes tout court. Mais ensuite, même à cet égard, comme le remarquera plus tard Michelle Perrot, « un certain silence s'était instauré " ${ }^{38}$. C'est pourquoi l'organisation, en novembre 1992, du colloque Femmes dans la cité sous l'impulsion de Michèle Riot-Sarcey et Jacqueline Lalouette, constitue une date importante. Ce colloque auquel participèrent très majoritairement de jeunes chercheuses et qui fut entièrement dirigé par des femmes, fit également une part à peu près égale à une histoire des femmes de type traditionnel et à celle qui prenait pour axe de recherche la notion de genre ${ }^{39}$. Le volume des actes une fois paru, eut un certain succès. Cette avancée assez tardive dans le domaine du « genre » devait se confirmer par la suite dans le contenu de la Revue. Sous l'impulsion d'Alain Corbin notamment, des sujets très originaux, l'exil, l'incendie, le silence ont fait l'objet de numéros spéciaux

domaines en revanche, l'intérêt de la société apparaît beaucoup plus faible. C'est particulièrement sensible sur la question de l'Europe qui n'a été abordée que dans un numéro spécial d'ailleurs modeste (53 pages), et d'une façon générale en matière internationale bien que le cent cinquantenaire ait permis de rattraper un peu le retard. C'est encore plus vrai sur une question où la recherche a fait pourtant pendant les dernières années d'énormes progrès, c'est à dire sur la question de la protection sociale au sens large. Le bilan est un peu meilleur sur les questions culturelles, domaine immense, abordé à la fois dans le grand colloque sur l'Usage de l'image au XIX ${ }^{e}$ siècle (1990) mené en collaboration avec la Société des études romantiques et le musée d'Orsay, et dans un numéro spécial de la revue ${ }^{40}$ et suivi avec attention dans les recensions bibliographiques de celle-ci. La proximité de la Société des études romantiques qui fait un excellent travail en la matière, incite en outre à ne pas développer une concurrence inamicale sur ce terrain. Enfin, il faut signaler les efforts qu'a accomplis notre société 
dans la dernière période pour aborder des sujets plus théoriques, ce fut le cas dans l'essai encore inachevé de Repenser le XIXe siècle mené en collaboration avec la Société des études romantiques et aussi dans notre journée d'études sur Le Temps et les Historiens dont les actes parurent en 2002.

Quelles conclusions tirer de ce rapide exposé ? Tout d'abord, retenons qu'il ne porte que sur les publications au sens défini ci-dessus, et que cette vision est donc partielle. Une approche qui comprendrait aussi la totalité des articles de la revue, mais qui aurait été beaucoup plus lourde à mener à bien, apporterait sans doute quelques nuances. Malgré ses faiblesses, le bilan de l'activité éditoriale de la société sur un siècle est assez impressionnant et représente une masse considérable de matériaux historiques et de réflexions, qui ne sont sans doute pas toujours assez bien utilisés par les jeunes chercheurs.

Nous nous sommes attaché à étudier en tant que telle la politique éditoriale parce qu'il nous semblait intéressant de faire apparaître comment celle-ci est déterminée à la fois par la nature même de l'éditeur (c'est-à-dire une société savante, qui n'a eu qu'occasionnellement des appuis institutionnels, ce qui nous a toujours condamné à des ressources essentiellement précaires), et par un contexte plus vaste, c'est-à-dire l'évolution générale des structures de la recherche en sciences humaines, celle aussi des formes concrètes, elles aussi évolutives, de cette même recherche et qu'une société savante épouse naturellement, évolution qui nous a fait passer, pour retenir l'essentiel, de la publication de documents aux recueils thématiques, puis aux actes de colloque et numéros spéciaux de revue. Nous n'avons guère abordé la diffusion de ces publications, d'ailleurs mal connue, mais vraisemblablement toujours assez faible. Constatons pour finir que si nous sommes récemment entrés dans l'ère d'Internet, pour l'instant, celle-ci n'a que peu d'influence sur notre activité éditoriale.

Quant à l'évolution de nos thèmes de recherche, elle a suivi évidemment l'évolution de la conjoncture historiographique, mais avec des inflexions particulières. Si la diversification des thèmes d'étude qui correspond à une tendance générale de l'histoire surtout à partir des années 1960 est sensible, le recul de l'histoire sociale et politique très dominante au départ, n’a été que limité, la percée de l'histoire économique dans les années 1950 a été et demeure faible. L'évolution interne de notre société n'a pas empêché une certaine fidélité aux orientations originelles, enrichie d'ailleurs par de nouvelles curiosités. Enfin, malgré quelques efforts d'ouverture sur le monde étranger, notre domaine d'étude est resté dans l'ensemble très franco-français.

Aujourd'hui, en matière de publications, notre société est confrontée à des exigences à la fois anciennes et nouvelles : il lui faut à la fois maintenir son existence en tant que société savante autonome et pour cela développer le nombre de ses adhérents qui sont aussi des clients potentiels, mais il lui faut aussi s'appuyer plus solidement sur des structures institutionnelles ou commerciales pour éditer et diffuser, conserver ses travaux, et aussi exploiter les nouvelles possibilités offertes par Internet. Quant aux thèmes de recherche, ils continueront naturellement à évoluer et de façon sans doute peu prévisible, mais il est sûrement souhaitable de maintenir un équilibre entre ceux qui ont fait le succès historique de la société et qui dessinent sa personnalité, et les explorations nouvelles, elles aussi indispensables pour renouveler son image et la maintenir en phase avec la recherche vivante. 


\section{ANNEXES}

Liste des publications de la Société d'histoire de la révolution de 1848 (ouvrages et numéros spéciaux de revue) (1908-2004)

Les volumes 1 à 24 font partie d'une collection intitulée « Bibliothèque d'histoire de la révolution de 1848 ».

1. Procès-Verbaux du Comité du travail à l'Assemblée Constituante de 1848, Société nouvelle de Librairie et d'édition, 1908, in 8 XII-329 p. Cote BNF : MFICHE 8-Lb52-103 (1).

2. Mémoires de l'ouvrier François Leblanc, adjoint au maire de Monville [Seine-Inférieure] en 1848. Projet d'Adolphe Peynaud [...] sur la crise industrielle de 1848 à Rouen.

Documents édités par Alphonse-Marius Gossez, Paris, Cornély, 1907-8, in $8^{\circ}, 72$ p. Cote BNF : 8 Lb52 103.

3. Hubert Bourgin, Victor Considerant, son œuvre, Lyon, imp. Réunies, 1909, in $4^{\circ}, 128 \mathrm{p}$. Cote BNF : 4 Ln 2780729.

4. François Dutacq, Histoire politique de Lyon pendant la révolution de 1848 (25 février-15 juillet), Paris Cornély, 1910, in 8², 459 p., pl. Cote BNF : 8 -Lk7 -37371.

5. Hippolyte Monin, François Désiré Bancel, représentant de la Drôme, proscrit, professeur à Bruxelles, député de Paris (1822-1871), d'après ses lettres inédites, Paris, Cornély, 1911, in $8^{\circ}$, 148 p. Cote BNF : MFICHE 8-Lb52-103 (5).

6. Victor Fleury, Le poète George Herwegh 1817-1875, Paris, Cornély, 1911, grand in $8^{\circ}$, XII-398 p. Cote BNF : 8-Lb52- 103(6).

7. Robert Pimienta, La propagande bonapartiste en 1848, Paris, Cornély, grand in $8^{\circ}, 1911$, 128 p. Cote BNF : 4- Lb52- 119.

8. Félix Ponteil, La Crise alimentaire dans le Bas-Rhin en 1847 d'après des documents inédits, Rennes imp. Réunies, s d., in $8^{\circ}, 141$ p. (extrait de La Révolution de 1848, année 1925-26). Cote BNF : 4 -Lk4 -3845.

9. Gabriel Perreux, Arbois première cité républicaine de France, étude locale sur les révolutions du XIX' siècle, Paris, Rieder, 1932, in 8 46 p. Cote BNF : 8-Lb52-103 (9).

10. Souvenirs d'un soldat sur la campagne de 1823 en Espagne, édités par Alphonse-Marius Gossez, Paris, Rieder, 1933, in 8, 94 p. Cote BNF : MFICHE 8 -Lb52 -103 (10).

11. Donald McKay, Un imprimé « perdu » sur la dissolution des Ateliers Nationaux de 1848, Paris, Rieder, 1933, in $8^{\circ}, 32$ p. Même cote que le précédent.

12. M.M. [Marie-Madeleine] Kahan-Rabecq,. La crise des subsistances dans le Haut-Rhin, à la veille de la révolution de 1848 et la « fête des boulangers » à Mulhouse en juin 1847, Rieder, 1937, in $8^{\circ}, 48$ p. Cote Bibliothèque universitaire Strasbourg : D 121984 (12).

13. Souvenirs du colonel Vergnaud, officier d'artillerie 1791-1885. Analyse et extraits, édités par Alphone-Marius Gossez, fascicules I et II, Paris, Rieder, 1937, 188 et 104 p., portrait. Cote BNF : 8 - Lb52- 103 (13). 
14. Alphone-Marius Gossez, L'ouvrier lillois du textile et sa situation sociale autour de 1848. Avec L'ouvrier manufacturier dans la société conservatrice, par Alphonse Bianchi, Paris, Rieder, 1938 , in $8^{\circ}, 32$ p. Même cote que le précédent.

[À l'occasion du centenaire de 1848, la Société est impliquée dans de nombreuses publications dont elle n'est pas la seule initiatrice. On en trouvera la liste dans l'encadré qui figure à la fin de ce document]

15. Études de Pierre Angrand, Georges Bourgin, Abel Chatelain, M. Faucheux et L. Morauzeau, Henri Gontard, Rémi Gossez, Paul Guichonnet, Edith Thomas, Nancy, imprimerie Thomas, 1953 , in $8^{\circ}, 164 \mathrm{p}$.

16. Études de André Armengaud, Maurice Dommanget, Jacques Droz, Henri Dubief, Jeanne Gaillard. Jacques Godechot, A. Hamelin, Jean Vidalenc, Nancy, imprimerie Thomas, 1954, in $8^{\circ}, 150 \mathrm{p}$.

17. Lise Dubief, Table alphabétique et méthodique des publications de la Société d'histoire de la révolution de 1848, in $8^{\circ}$, La Roche-sur-Yon, imprimerie centrale de l'Ouest, 1957, 44 p.

18. Études de Jacques Bouillon, Pierre Chalmin, Suzanne Coquerelle, Raoul Girardet, Rémi Gossez, Nicole Villa sur L'Armée et la Seconde République, Nancy, imprimerie centrale de l'Ouest, in $8^{\circ} 1955,159$ p. Cote BNF : 8- Lb52 -103 (18).

19. Études sous la direction d'Ernest Labrousse, Aspects de la crise et de la dépression de l'économie française au milieu du XIXe siècle 1846-1851, La Roche-sur-Yon, imprimerie centrale de l'Ouest, 1956, in $8^{\circ}$, XXIV-356 p. [contributions de Maurice Agulhon, Paul Bois, A. Chanut, F.P. Codaccioni, Gabriel Désert, Pierre Deyon, François-Georges Dreyfus, Marcel Gillet, Paul Gonnet, Rémi Gossez, Pierre Guiral, Ernest Labrousse, F. Lentacker, L. Machu, Michelle Perrot (carte).Cote BNF : 8 Lb52- 103 (19).

20. Le Choléra, la première épidémie du XIX siècle. Étude collective présentée par Louis Chevalier [dir.], La Roche-sur-Yon, imprimerie centrale de l'Ouest, 1958, in $8^{\circ}$, XVIII-189 p., fig., plans [contributions de Louis Chevalier, Monique Dineur, Charles Engrand, Dr Freour, Pierre Guiral, M.V. Netchkina, K.V. Sivkov, A.L. Sidorov, David Eversley]. Cote BNF : 8 -Lb52 -103 (20).

21. Les élections de 1869, études présentées par Louis Girard, Paris, Librairie Marcel Rivière, 1960, in $8^{\circ}$, XXXII-215 p., cartes, plans [contributions de Rémi Gossez, J. Bertin, Jacques Rougerie, Jeanne Gaillard, Antoine Olivesi, M. Faucheux, Pierre Lévèque, Guy Palmade]. Cote BNF : 8- Lb52-103 (21).

22. Réaction et suffrage universel en France et en Allemagne 1848-1850, Études présentées par Jacques Droz, Paris, Librairie Marcel Rivière, 1963, in 8 ${ }^{\circ}$ IV-180 p. [contributions de Jacques Droz, G. Schilfert, Robert Balland]. Cote BNF : 8 -Lb52-103 (22).

23. La Presse ouvrière 1819-1850, Angleterre, États-Unis, France, Belgique, Allemagne, Italie, Allemagne, Tchécoslovaquie, Hongrie, Études présentées par Jacques Godechot, Société d'histoire de la révolution de 1848, 1966, in 8 312 p. Cote BNF : 8- Lb52-103 (23).

24. Rémi Gossez, Les Ouvriers de Paris, I, L'organisation 1848-1851, Paris, Société d'Histoire de la révolution de 1848, 1968, in $8^{\circ}, 447$ p., plan, Cote BNF : 8 -Lb52 -103 (24).

Avec ce volume prend fin la Bibliothèque de la révolution de 1848 
25. Annales historiques de la Révolution française, juillet-septembre 1977, numéro spécial sans titre consacré à la question des prisons. Textes de Maurice Agulhon, Michelle Perrot, Jacques Léonard, Nicole Castan, Catherine Duprat, Jean-Jacques Darmon, André Zysberg. Numéro reproduit avec des textes complémentaires, notamment de Michel Foucault, Maurice Agulhon, Jacques Valette, Jean Lebrun, sous la direction de Michelle.Perrot sous le titre L'Impossible prison. Recherches sur le système pénitentiaire au XIX ${ }^{e}$ siècle, Paris, Édition du Seuil, L’Univers historique, 1980, 317 p. Cote BNF : 944.06/ 305 Perr.i.

26. 1848. Les utopisme sociaux, textes rassemblés par Jacques Valette, préface de Maurice Agulhon CDU-SEDES, 1981, 290 p.

27. Blanqui et les Blanquistes, Actes du Colloque Blanqui $\left(1^{\mathrm{er}}, 2,3\right.$ octobre 1981$)$ [centième anniversaire de la mort de Blanqui]. Préface de Philippe Vigier, Paris, CDU-SEDES, 1986, $292 \mathrm{p}$.

28. Maintien de l'ordre et police en France et en Europe au XIX ${ }^{e}$ siècle. Présentation de Philippe Vigier, Introduction d'Alain Faure. Actes du Colloque des 8, 9 et 10 décembre 1983, Paris, Éditions Créaphis, 1987, 412 p.

29. Numéro spécial de la Revue 1848, Révolutions et mutations au XIXe siècle : " Histoires de Centenaires ou le devenir des Révolutions [1830, 1848, 1870 et 1871] », n 5, 1989, présentation de Jean-Claude Caron, pp. 19-126.

30. Répression et prison politique en France et en Europe au XIX $X^{e}$ siècle, présentation de Philippe Vigier, introduction d'Alain Faure. Actes du colloque des 6, 7 et 8 mars 1986, Paris, Éditions Créaphis, 1990, 327 p.

31. "Sentiment et espace européens au XIX ${ }^{\mathrm{e}}$ siècle », 1848. Révolutions et mutations au XIX siècle [ci dessous 1848 R\&M], nº 7,1991, pp. 19-72.

32. «Jeunesses au XIX ${ }^{\mathrm{e}}$ siècle ", 1848 R\&M, n 8, 1992, pp. 19-99.

33. Le XIX $X^{e}$ siècle et la Révolution française, préface de Maurice Agulhon, introduction d'Alain Faure. Actes du colloque des 13 et 14 octobre 1989, Paris, Éditions Créaphis, 1992, 429 p.

34. Stéphane Michaud, Jean Yves Mollier, Nicole Savy [dir.], Usages de l'image au XIX ${ }^{e}$ siècle. Préface de Maurice Agulhon. Actes du colloque organisé par la Société des études romantiques et dix-neuviémistes, la Société d'histoire de la révolution de 1848, et le Musée d'Orsay, 24 au 26 octobre 1990, Paris, Éditions Créaphis, 1992, 257 p.

35. « Utopies au XIX siècle », 1848 R\&M, nº 9, 1993, pp. 21-56.

36. « Le silence au XIX ${ }^{\mathrm{e}}$ siècle »,1848 R\&M, n 10, 1994, présentation d'Alain Corbin, pp. 23-69.

37. « L'exil », 1848 R\&M, n 11, 1995, présentation d'Alain Corbin, pp. 11-76

38. «L'incendie », Revue d'Histoire du XIX ${ }^{e}$ siècle [ci-dessous RH19], n 12, 1996, pp. 7-56.

39. Jean-Claude Caron, Alain Corbin, Michèle Riot-Sarcey, Rosemonde Sanson [dir.], « Repenser le XIX ${ }^{\mathrm{e}}$ siècle », RH19, n 13, 1996, pp. 9-112.

40. Alain Corbin, Jacqueline Lalouette, Michèle Riot-Sarcey [dir.], Femmes dans la cité, 1815-1871, Actes du colloque des 26 et 27 novembre 1992, Paris, Éditions Créaphis, 1997, $574 \mathrm{p}$. 
41. Alain Corbin, Jean Marie Mayeur [dir.], La Barricade, Actes du Colloque organisé par le centre de recherches en histoire du XIX ${ }^{\mathrm{e}}$ siècle (Universités de Paris I-Paris IV) et la Société d'histoire de la révolution de 1848, 17, 18 et 19 mai 1995, Paris, Publications de la Sorbonne, 1997, $522 \mathrm{p}$.

42. Francis Démier, Jean-Luc Mayaud [dir.], « Cinquante ans de recherche sur 1848 », RH19 , n 14, 1997/1, pp. 11-199.

43. Jean-Claude Caron, Michèle Riot-Sarcey [dir.], « 1848, Nouveaux Regards », RH19, $\mathrm{n}^{\circ}$ 15, 1997/2, pp. 5-88.

44. Pierre Lenoël, Jean Jacques Yvorel [dir.], « 1848, Un modèle politique à l'épreuve », RH19, $\mathrm{n}^{\circ} 16,1998 / 1$, pp. 9-159.

45. Jean -Yves Mollier [dir.], « Les foules au XIX siècle », RH19, $\mathrm{n}^{\circ}$ 17, 1998/2, pp. 9-76.

46. Dominique Kalifa [dir.], « Aspects de la production culturelle au XIX ${ }^{\mathrm{e}}$ siècle », RH19, $\mathrm{n}$ • 19, 1999/2, pp. 7-116.

47. Raymond Huard [dir.], « Autour de décembre 1851 », RH19, n² 22, 2001/1, pp. 9-146.

48. PatrickVerley, Jean-Luc Mayaud [dir.], « Nouvelles approches en histoire économique », RH19, n²3, 2001/1, pp. 7-227.

49. Jean-Luc Mayaud [dir.], 1848 Actes du colloque international du cent cinquantenaire tenu à l'Assemblée nationale, les 23-25 février 1998, Paris, Éditions Créaphis, 2002, 583 p.

50. Sylvie Aprile, Jean-Claude Caron, Michèle Riot-Sarcey [dir.], « Le temps et les historiens ", RH19, n²5, 2002/2, pp. 5-202.

51. Sylvie Aprile, Nathalie Bayon, Laurent Clavier, Louis Hincker, Jean-Luc Mayaud [dir.], Comment meurt une République, autour du 2 décembre 1851, Actes du colloque de Lyon, 28, 29, 30 novembre et $1^{\text {er }}$ décembre 2001, Paris, Éditions Créaphis, 2004, 459 p.

52. Jacqueline Lalouette, Michèle Riot-Sarcey [dir.], « Religion, politique et culture au XIX ${ }^{\mathrm{e}}$ siècle ", RH19, n 28, 2004, pp. 9-173.

La société de 1848 et le centenaire de la révolution de 1848

À l'occasion du centenaire de 1848, la société est impliquée, en collaboration avec d'autres organismes, dans la publication de nombreux ouvrages de trois catégories :

1. Documents

Procès verbaux du gouvernement provisoire et de la commission du pouvoir exécutif, 24 février22 juin 1848, avant-propos de Charles-Hippolyte Pouthas, Annotations et Index par Albert Soboul, Paris, Imprimerie nationale, 1950, grand in 8, XIX-432 p. Cote BNF : 4 Lb52 140.

2. Actes de colloque

Comité français des Sciences historiques, Actes du Congrès historique du centenaire de la révolution de 1848, préface de Robert Fawtier, Paris, Presses universitaires de France, 1948, XIII-433 p. Cote BNF : 4 G 2539 ou Microfiche M 21360.

3. Ouvrages de vulgarisation :

Collection du centenaire de la révolution de $1848: 23$ ouvrages de format $19 \times 12 \mathrm{~cm}$, 76 à 80 pages. Paris, Presses universitaires de France, 1948, Cote BNF : Microfiche 8297 (1 à 23).

1. Edith Thomas, Les femmes de 1848. 
2. Sylvain Molinier, Blanqui.

3. Jean Cassou, Le Quarante-huitard.

4. Jean Bruhat, Les Journées de février 1848.

5. Armand Cuvillier, P.G.B. Buchez et les origines du socialisme chrétien.

6. Emile Tersen, Le gouvernement provisoire et l'Europe, 25 février-12 mai 1848.

7. Paul Bastid, L'avènement du suffrage universel.

8. Robert Schnerb, Ledru-Rollin.

9. Pierre Chaunu, Eugène Sue et la Seconde République.

10. Charles Schmidt, Des ateliers nationaux aux barricades de juin.

11. Jean-Louis Puech et Edouard Dolléans, Proudhon et la révolution de 1848.

12. Auguste Cornu, Karl Marx et la révolution de 1848.

13. Jean Vidalenc, Louis Blanc 1811-1882.

14. Pierre Angrand, Cabet.

15. Gaston Martin, L'abolition de l'esclavage, 27 avril 1848.

16. Félix Armand, Les Fouriéristes et les luttes révolutionnaires de 1848 à 1851.

17. Henri Guillemin, Lamartine en 1848.

18. Justin Godart, À Lyon en 1848, les Voraces.

19. Jean Pommier, Les écrivains devant la révolution de 1848 : Lamartine, Hugo, Lamennais, George Sand, Michelet, Béranger.

20. Paul Cunisset-Carnot, Hippolyte Carnot et le ministère de l'Instruction publique de la Seconde République.

21. René Rémond, Lamennais et la démocratie.

22. Georges Duveau, Raspail.

23. Gaston Monnerville : Commémoration du centenaire de l'abolition de l'esclavage, discours prononcé à la Sorbonne le 27 avril 1848 par Gaston Monnerville, Léopold Sédar Senghor et Aimé Césaire.

\section{NOTES}

1.. Nous remercions les membres de notre société qui ont amicalement répondu à nos demandes d'information sur des points précis et en particulier Jacqueline Lalouette, Michelle Perrot, Michèle Riot-Sarcey, Maurice Agulhon, Alain Faure et Jean-Yves Mollier. 2.. On trouvera la liste de ces publications ainsi que toutes les indications bibliographiques concernant ces volumes dans la liste qui figure à la fin de cet article. Il n'est pas aussi facile qu'on pourrait le croire d'accéder à ces ouvrages, en particulier pour la période la plus ancienne. Même si la Bibliothèque nationale de France possède une collection assez complète, il manque au moins un volume ( $\left.n^{\circ} 12\right)$. Un autre $\left(n^{\circ} 11\right)$ qui ne 
figure pas au catalogue, est néanmoins dans les collections parce qu'il est joint à un autre ouvrage. Pour le numéro 2 , la cote donnée est en réalité inutilisable.

3. Rapport de Georges RENARD à l'assemblée générale du 24 février 1906, dans Bulletin de la société d'histoire de la Révolution de 1848, 1906-1907 pp. 58-59.

4.. Rapport de Georges RENARD à l'assemblée générale du 24 février 1907, dans Bulletin, 1907-1908, p. 6.

5.. Ibidem.

6.. Pour la référence de ce livre comme pour tous les ouvrages publiés par la Société qu'on citera par la suite, on se reportera à la liste détaillée fournie à la fin de cet article.

7.. Rapport d'Hippolyte MOYSSET, secrétaire général à l'assemblée générale de 1910, dans Bulletin, 1910-1911, p. 4. En 1909, la parution du Mémorial avait déjà été annoncée pour octobre, dans Bulletin, 1919-1910, pp. 2-3.

8.. Rapport d'Hippolyte MOYSSET à l'assemblée générale de 1911, dans Bulletin, 1911-1912, p. 6.

9.. Bulletin,1909-1910, pp. 20-33. Il s'agit du récit du 24 février 1848.

10.. Renseignement fourni par Jean-Yves Mollier (lettre du 27 juin 2004).

11.. En 1947, Emile TERSEN avait surtout travaillé sur l'abolition de l'esclavage et sur les relations extérieures du gouvernement provisoire. Il publiera en 1957 un Quarante- huit, Paris, Club français du livre, $284 \mathrm{p}$.

12. Sur ces moments de l'histoire de la Société de 1848, nous renvoyons aux deux contributions données par Rémi Gossez à 1848, Mutations et Révolutions au XIXe siècle, «Éléments pour un historique de la société d'histoire de la Révolution de 1848 et des révolutions du XIX ${ }^{\mathrm{e}}$ siècle, I, 1904-1940 », 1985, pp. 17-30, et « La société de 1848 (1945-1980) », 1986, pp. 13-22.

13. Voir la liste ci-dessous.

14.. Actes du Congrès historique du centenaire de la Révolution de 1848, Paris, Presses universitaires de France, 1948, pp. 1-20.

15.. « 1848-1948. Les printemps des peuples. Le centenaire en France. », entretien de Philippe Vigier et Rémi Gossez, avec Jean-Claude Caron et Pierre Lenoël, dans 1848, Mutations et Révolutions au XIX ${ }^{e}$ siècle, $\mathrm{n}^{\circ}$ 5, 1989, p. 51.

16.. Rémi GOSSEZ, «La société de 1848 », art. cité, 1986, p. 19.

17.. Ibidem, p. 18.

18.. Ibidem, p. 19.

19. Le volume « Chômage et bas-fonds » est annoncé à la fin de La presse ouvrière. Pour le second, voir Rémi GOSSEZ, art. cité, 1986, p. 19.

20. Lettre de Maurice Agulhon à l'auteur, 23 mars 1975.

21. Maurice AGULHON et Albert SOBOUL, "À nos lecteurs ", dans Annales historiques de la Révolution française, $\mathrm{n}^{\circ} 222$, octobre-décembre 1975, p. 497.

22. Nous suivons ici le témoignage téléphonique détaillé de Michelle Perrot (début juillet 2004) et celui, écrit, de Maurice Agulhon, 28 août 2004.

23. Jacques LÉONARD «L'historien et le philosophe », dans Annales historiques de la Révolution française, $\mathrm{n}^{\circ}$ 228, juillet-septembre 1977, pp. 163-181.

24.. « La poussière et le nuage », dans L'impossible prison, Paris, Éditions du Seuil, 1980, pp. 29-39.

25.. Rémi GOSSEZ, art. cité, 1986, p. 20.

26. Socialisme utopique et idée coloniale, Jules Duval, 1814-1870, Université de Paris I, 1975.

27.. Allocation d'ouverture du colloque dans Blanqui et les Blanquistes, p. 10. 
28. Créaphis fut fondé en 1982 dans le sud de la Drôme, d'abord en association, autour du site de Saint-Restitut, avec un projet scientifique orienté vers l'étude de la pierre et de son histoire. À partir de 1987, son activité éditoriale se diversifia vers d'autres domaines (histoire sociale et politique, architecture et habitat, photographie).

29.. Préface à l'ouvrage, p. 1.

30.. Jacques DROZ, « L'influence de Marx en Allemagne pendant la révolution de 1848 », dans Études, 1954, pp. 3-16.

31.. Actes, ouv. cité, p. 31-46.

32. Rémi GOSSEZ, art. cité, 1986, p. 21.

33.. « Une féministe de 1848, Jeanne Deroin, dans La Révolution de 1848, mars 1907-février 1908, pp. 317-355.

34.. « Les républicaines du Second Empire ", dans 1848 et les révolutions du XIX ${ }^{e}$ siècle, printemps 1947-automne 1948, pp. 38-55.

35.. "Le rôle des femmes dans la Commune de Paris, 1871 », dans 1848 et les révolutions du XIX ${ }^{e}$ siècle, février 1949, pp. 15-29.

36.. Lettres de Pauline Roland à Gustave Lefrançais, dans Études, 1953, pp. 136-142.

37.. La presse ouvrière, 1966, pp. 88-122.

38. Préface à Femmes dans la Cité, p. 15.

39.. Témoignages de Michèle Riot-Sarcey et Jacqueline Lalouette à l'auteur, 4 juillet et 7 septembre 2004.

40.. Revue d'histoire du XIX $X^{e}$ siècle, $\mathrm{n}^{\circ}$ 19, 1999/2, sous la direction de Dominique Kalifa, pp. 7-116.

\section{RÉSUMÉS}

En 1906, la Société d'histoire de la Révolution de 1848 décida de procéder à des publications qui s'ajouteraient à son bulletin. Depuis cette date, la politique de publication de la Société a changé plusieurs fois, mais au total, son effort a abouti à la publication d'une cinquantaine de volumes. L'article montre comment les publications, qui étaient au départ consacrées surtout à des documents d'histoire sociale évoluèrent au cours du siècle, et comment on passa d'abord à des livres sur un sujet précis puis à des recueils d'études. Plus récemment, la Société a privilégié deux types de publications ; numéros spéciaux de la Revue et actes de colloques. La seconde partie de l'étude est consacrée à l'évolution de thèmes de recherche révélée par les publications .Celle-ci s'est opérée en fonction de l'évolution générale de la recherche historique, mais aussi de la conjoncture politique et sociale, d'occasions plus circonstancielles (le centenaire de 1848) ou de l'influence de tel président.

A century of publications by the "Société d'Histoire de la Révolution de 1848 ». The editorial policy and the evolution of research themes

In 1906, the "Société d'Histoire de la Révolution de 1848 » decided to publish other works in addition to its Bulletin. Since then, the Society's editorial policy has changed several times. But all in all, this effort has resulted in the publication of some fifty volumes. This paper shows how the publications, which were initially mainly social history documents, evolved to become successively books on a single subject, and then collections of studies. More recently, the society 
has favoured two types of publications: special issues of the Journal, and conference proceedings. The second part of the paper deals with the evolution of research themes as shown in the publications, due in part to the general evolution of historical research, but also to the political and social situation or to various events such as the centenary of 1848 , or the influence of a particular president.

\section{AUTEUR}

\section{RAYMOND HUARD}

Professeur émérite à l'Université Paul Valéry-Montpellier 